\title{
Serviceability Assessment for Cascading Failures in Water Distribution Network under Seismic Scenario
}

\author{
Qing Shuang, ${ }^{1,2}$ Yisheng Liu, ${ }^{1,2}$ Jing Liu, ${ }^{1,2}$ and Qigang Chen ${ }^{3}$ \\ ${ }^{1}$ Department of Construction Management, School of Economics and Management, Beijing Jiaotong University, \\ Beijing 100044, China \\ ${ }^{2}$ Beijing Center for Industrial Security and Development Research, Beijing 100044, China \\ ${ }^{3}$ School of Civil Engineering, Beijing Jiaotong University, Beijing 100044, China \\ Correspondence should be addressed to Qing Shuang; qings@bjtu.edu.cn
}

Received 29 November 2015; Revised 23 March 2016; Accepted 31 March 2016

Academic Editor: Stefano de Miranda

Copyright (C) 2016 Qing Shuang et al. This is an open access article distributed under the Creative Commons Attribution License, which permits unrestricted use, distribution, and reproduction in any medium, provided the original work is properly cited.

\begin{abstract}
The stability of water service is a hot point in industrial production, public safety, and academic research. The paper establishes a service evaluation model for the water distribution network (WDN). The serviceability is measured in three aspects: (1) the functionality of structural components under disaster environment; (2) the recognition of cascading failure process; and (3) the calculation of system reliability. The node and edge failures in WDN are interrelated under seismic excitations. The cascading failure process is provided with the balance of water supply and demand. The matrix-based system reliability (MSR) method is used to represent the system events and calculate the nonfailure probability. An example is used to illustrate the proposed method. The cascading failure processes with different node failures are simulated. The serviceability is analyzed. The critical node can be identified. The result shows that the aged network has a greater influence on the system service under seismic scenario. The maintenance could improve the antidisaster ability of WDN. Priority should be given to controlling the time between the initial failure and the first secondary failure, for taking postdisaster emergency measures within this time period can largely cut down the spread of cascade effect in the whole WDN.
\end{abstract}

\section{Introduction}

Water distribution network (WDN) is a basic component in civil infrastructure systems. Its stability and reliability are important to ensure industrial production and public safety. Nowadays, WDN has developed into a large-scale network with thousands of pipes and nodes [1]. In such circumstance, how to design, improve, monitor, and repair the components efficiently in WDN becomes a critical problem in risk and reliability analysis. However, such analyses are often challenging due to complex network topology [2], components interdependency [3], and hydraulic calculation. Component failures may lead to the cascade effects and secondary failures under seismic scenario $[4,5]$. This cascade reaction will prolong the repair cycle and lead to economic losses [6]. Therefore, it is necessary to quantify the effects of such cascading failures, to develop a system reliability evaluation method under natural hazards and to further analyze the serviceability of the WDN.

Studies on the WDN reliability under seismic scenario have been attracting extensive attention. Shinozuka et al. [7] analyzed the WDN of Memphis and evaluated the consumer demand under seismic scenario. Hwang et al. [8] analyzed the damage of pipes and the soil liquefaction under seismic scenario by using GIS technology. The serviceability was simulated by using Monte Carlo method. Regarding the power system as the backup of WDN, Adachi and Ellingwood [9] made serviceability analysis on the interaction influence of the power system and WDN under seismic scenario. Brink et al. [10] evaluated and compared the WDN emergency measures of Los Angeles Hydropower Board under seismic scenario. However, the studies above are concentrated on the reliability and serviceability of pipe network under seismic scenario, instead of considering the effect of cascade. 
The identification of critical nodes is an important aspect of the system design and antidisaster ability of urban infrastructure [11]. Research shows that the failure of the critical nodes or edges may trigger the disastrous consequences, such as widespread avalanche and complete collapse [12, 13]. The research is focused on cascading failure of complex network, which is to find out the critical nodes of the network. Furthermore, an important step in WDN evaluation under seismic scenario is to identify the nodes which influence the serviceability seriously $[14,15]$. Hence, to WDN, analyzing the serviceability of the system and identifying the critical nodes are crucial in guaranteeing the urban safety.

To analyze the serviceability in WDN, technologies from complex network and system reliability can be used. The serviceability can be measured in three perspectives: (1) functionality of structural components under disaster environment; (2) recognition of cascading failure process; and (3) reliability calculation and further evaluation of the system serviceability.

This paper studies the serviceability of WDN in cascading failure caused by seismic action. Three factors, that is, the seismic attenuation, cascading failure, and reliability of water supply, are taken into consideration. The cascading failure process is provided with the balancing of water supply and demand. The node and edge failures in WDN are interrelated under seismic excitations. The matrix-based system reliability (MSR) method is used to represent the system events and calculate the nonfailure probability. The influence of serviceability is evaluated with system reliability and cascading failure process. This method is applied to WDN. On the consideration of the antiseismic reliability of the single component and the whole system, the paper analyzes the influence of the network tolerance parameter on system serviceability. The method adopted in the paper can help the decision-makers to identify the critical nodes, resist the possible widespread network failure under disasters, and improve the WDN serviceability.

\section{Simulation of the Cascading Failure Process in WDN}

2.1. Cascading Failure Model. The load distribution on the network is determined by many factors. The load can be the material, information, and energy [12]. In many entity networks, the load is transmitted along the edges based on the strategy of shortest path. The nodal capacity is the maximum load that a node can bear. As the goal of system optimization is to maximize the system operation effects with the minimum cost, it can be assumed that there exists a proportional relation between the bearing capacity and the initial load:

$$
C=(1+\alpha) L
$$

where $C$ is the node capacity; $L$ is the node load; $\alpha$ is the tolerance parameter, $0 \leq \alpha \leq 1$. $\alpha$ is a tunable parameter [16]. It gives a way to control the strength of the capacity.

When the load is out of the capacity range, the node loses its functions and triggers the flow redistribution. The flow redistribution may lead to new failure nodes. This step-bystep process is a cascading failure [17-19]. Time $t$ is used to describe the cascading failure step. In the paper, $t$ is used for an algorithmic step. $t=0$ indicates no failure in WDN. $t=1$ describes the initial failure. $t=2,3, \ldots$ show the cascading failure process.

2.2. Cascading Failure Modelling in WDN. Whether a node in WDN can provide sufficient pressure and flow to the customers is the basic condition to judge the system operation. The service pressure is defined as the load. The nodal pressure is neither too high nor too low. In this scenario, a water node is operational if it can operate effectively and it functions as intended. It required that the nodal pressure is neither less than the design lowest nor higher than its capacity. The highest capacity is calculated by (1). After the initial node is attacked, if there are other nodes' loads out of its capacity range, new failure nodes are generated. The end condition is that no other node loses its function.

In order to measure the cascading failure in $\mathrm{WDN}$ after a particular node failed, it is assumed that the consequence of a failure node is completely damaged. Its adjacent edges quit from the system after the attack. Therefore, the failure is equivalent to deleting the node and its adjacent edges from the water network, which means the network's topology changed. With changes in the WDN topology structure, the water flow redistributed. Pressure of the operational nodes is calculated according to the laws of conservation of mass and energy. The Newton-Raphson method and node equations are involved to simulate the pressure throughout the network. Figure 1 shows the flowchart of cascading failure simulation in WDN.

\section{System Reliability}

3.1. Matrix-Based System Reliability Method. In recent year, the MSR method has been developed into an effective tool for calculating system reliability [20-22]. The principle is matrix-based calculation. This method has been proved to be capable of being applied to the components with statistical correlation, series system, and parallel system. Assume that a system has $n$-independent components. The $i$ th component has two states, that is, nonfailure or failure. Then, the sample space can be divided into $N=2^{n}$ mutually exclusive and collectively exhaustive (MECE) events which are represented by $e_{j}(j=1,2, \ldots, N)$. The system events can be described by event vector $c$. When $e_{j}$ represents a system event, the element value of $c$ corresponding to component $j$ is set as 1 ; otherwise it is set as 0 . Set the probability of $e_{j}$ as $p_{j}=P\left(e_{j}\right)$. According to the mutual exclusiveness of $e_{j}, P\left(E_{\text {sys }}\right)$ of $E_{\text {sys }}$ can be calculated by the sum of $p_{j}$. Therefore, the probability of any system event $\left(E_{\text {sys }}\right)$ can be calculated through the inner product of two vectors:

$$
P\left(E_{\text {sys }}\right)=\sum_{j: e_{j} \subseteq E_{\text {sys }}} p_{j}=c^{T} p,
$$

where $p$ contains the probability vector of $p_{j}, j=1, \ldots, N$. 


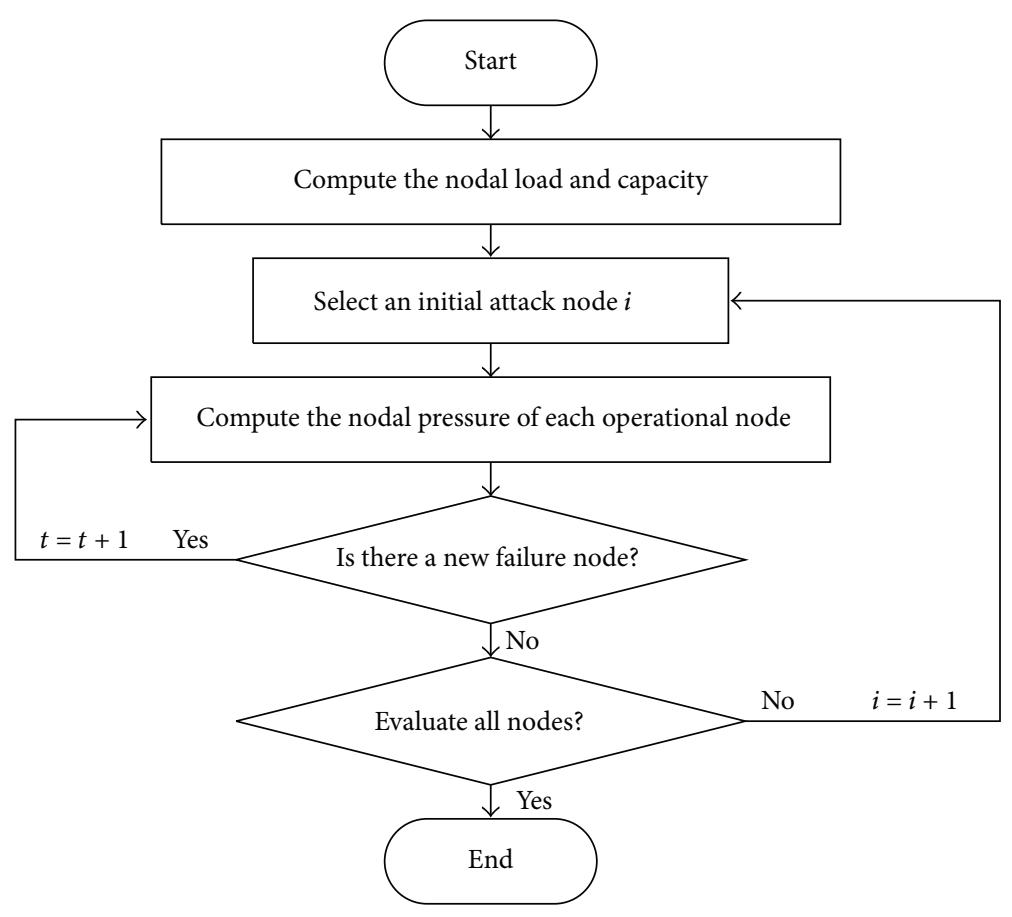

FIGURE 1: The flowchart of cascading failure simulation in WDN.

Let $c^{E}$ denote the event vector of event $E$; the vector of its complementary event $c^{\bar{E}}$ is

$$
c^{\bar{E}}=1-c^{E} .
$$

Then the intersection event and union event of events $E_{1}, E_{2}, \ldots, E_{n}$ are, respectively,

$$
\begin{aligned}
& c^{E_{1} \cap \cdots \cap E_{n}}=c^{E_{1}} \cdot * c^{E_{2}} \cdot * \cdots * c^{E_{n}} \\
& c^{E_{1} \cup \cdots \cup E_{n}}=1-\left(1-c^{E_{1}}\right) \cdot *\left(1-c^{E_{2}}\right) \cdot * \cdots \cdot *\left(1-c^{E_{n}}\right),
\end{aligned}
$$

where ".*" represents the multiplication between the elements of the matrix, which can be calculated by MATLAB. Equation (2) can be used to calculate the vector of any system event $E_{\text {sys }}$.

When the components are statistically correlated, (2) is not able to calculate the system probability. Instead, the minimum (maximum) probability of a system event can be established by linear programming (LP), so as to get the optimization boundary of the system event. Its mathematical expression is

$$
\begin{aligned}
\min (\max ) & c^{T} p \\
\text { s.t. } & a_{1} p=b_{1} \\
& a_{2} p \geq b_{2},
\end{aligned}
$$

where $b_{1}$ and $b_{2}$ are probability vectors; $a_{1}$ and $a_{2}$ are coefficient matrixes, whose rows correspond to the event vector of $b_{1}$ and $b_{2}$. The system probability interval obtained by LP method is the narrowest boundary which can be obtained from the given information [23]. Therefore, the system probability obtained by MSR method is the most accurate and effective estimation.
3.2. System Reliability under Seismic and Cascaded Scenario. Assume that the earthquake has caused the failure of a node on WDN. The nodal failure can probably trigger cascading failure. At this time, the new failures caused by cascading failure are independent of the earthquake. The subsystem reliability is measured by the operational nodes.

Use LP of MSR method to calculate the boundary of the system reliability because of the following: (1) the failure mode of WDN is internally correlated; a nodal failure can influence others; (2) different failure nodes lead to different failure paths due to the topological relations between nodes and pressure capacity; and (3) analyzing the reliability boundary of the disconnected nodes, Lim and Song [24] calculated the average of the upper and lower boundary. Results showed that the disconnection probability is overvalued when ignoring the spatial correlation. Therefore, LP method is adopted to calculate the reliability boundary. The average of the boundary is computed to avoid deviation. Let $c^{E_{i}}$ denote the probability of the $i$ th node antiseismic reliability. The objective function of the system reliability event can be calculated as

$$
E_{\text {sys }}=\bigcup_{k=1}^{K} \bigcap_{i \in C_{k}} E_{i} .
$$

The system reliability event in (5) is defined in terms of components $C_{k}, k=1, \ldots, K$, whose joint realizations constitute realizations of the system state $E_{\text {sys }} \cdot C_{k}$ denotes the set of components that constitute the $k$ th subsystem. The subsystem is a connected network constituted by the remaining operational nodes after cascading failures. Different nodes lead to different failure process. The subsystem reliabilities 


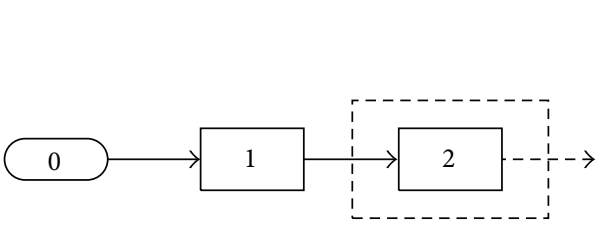

(a) Series system

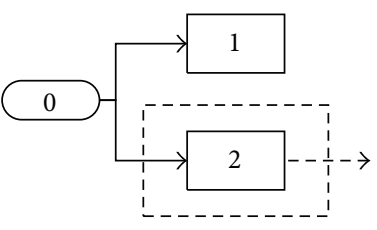

(b) Parallel system

Figure 2: Hypothetical system examples of series and parallel system.

constituted by the operational nodes at each time are also different. The reason is due to the hydraulic properties of the WDN and the topological structure of the pipe network.

Firstly, from the aspect of hydraulic properties, the WDN is a directed network. The upstream failure leads to the downstream failure which is directly connected with the upstream one. On the contrary, the downstream nodal failure does not result in the failure of the upstream nodes. The upstream nodes can supply water continually.

Secondly, from the aspect of network topology, when cascading failure occurred, (1) if the failure nodes correspond to the series position, the subsystem reliability increases. For example, in Figure 2(a), Node 0 is the supply node of the series system. The reliabilities of the three subnodes are $0.5,0.3$, and 0.3 , respectively. Assume that the nodes are independent; the system reliability is $E_{0} \cap E_{1} \cap E_{2}=0.5 \times 0.3 \times 0.3=$ 0.045 . If Node 2 at the tail end fails while Node 0 and Node 1 can still supply water (i.e., the pressures within the capacity), the system reliability increases to $E_{0} \cap E_{1}=0.5 \times$ $0.3=0.15$. (2) If the failure nodes correspond to the parallel position, the subsystem reliability decreases. For example, in Figure 2(b), Node 0 is the supply node of the parallel system. The reliabilities of the three subnodes are $0.5,0.3$, and 0.3 , respectively. The system reliability is $E_{0} \cap E_{1} \cup E_{2}=0.5 \times$ $(0.3+0.3)=0.3$. If Node 2 fails and it does not affect the supply of Node 0 and Node 1 , the system reliability decreases to $E_{0} \cap E_{1}=0.5 \times 0.3=0.15$.

3.3. Serviceability. The serviceability of WDN after earthquake contains two aspects: (1) the simulation of the failure state and (2) the analysis on the serviceability of the damaged network. After analyzing the influence of cascading failure and seismic action on WDN, it is necessary to measure the serviceability of the subsystem. Adachi and Ellingwood [9] developed the serviceability ratio. The serviceability ratio of WDN is related to the connectivity number of water distribution nodes. The serviceability ratio is given as

$$
S=\frac{\sum_{i=1}^{N}\left(\omega_{i} X_{i}\right)}{\sum_{i=1}^{N} \omega_{i}},
$$

where $S$ represents the serviceability ratio; $\omega$ is the weight; $X$ is the functionality of the infrastructure; $X=1$ indicates that the functionality of the infrastructure is effective; otherwise $X=0 ; N$ is the number of nodes in WDN.

The serviceability of WDN after earthquake should consider (1) the influence of seismic action on work nodes; (2) failure state caused by cascading failures; and (3) the serviceability of subsystem, comprehensively. Therefore, the aseismic reliability factor of the subsystem is taken into consideration based on (6). Equation (6) can be improved into (7). Equation (7) describes the serviceability of WDN after earthquake:

$$
S=\frac{\sum_{i=1}^{N}\left(\omega_{i} X_{i}\right)}{\sum_{i=1}^{N} \omega_{i}} P\left(E_{\text {sys }}^{t}\right),
$$

where $P\left(E_{\text {sys }}^{t}\right)$ is the system reliability event constituted by the work nodes at time $t$. The smaller the computed result of $\sum_{i=1}^{N} \omega_{i} X_{i}$ is, the fewer the function-effective infrastructures will be. The smaller the value of $P\left(E_{\text {sys }}^{t}\right)$ is, the lower the reliability probability of the system will be. As a result, $S$ is ranging between 0 and 1 ; the smaller $S$ is, the greater the serviceability loss caused by the cascading failure after earthquake will be.

\section{Data and Application Results}

The proposed method is illustrated by an example. The WDN [25] is shown in Figure 3. The pipe information includes pipe number, flow direction, pipe diameter, and pipe length. The node information includes node number, nodal demand, and ground elevation. Assume the minimum pressure is $6 \mathrm{~m}$ to ensure the basic operation.

The overall procedure is as follows:

Step 1 (network information)

(1) Provide WDN topology structure.

(2) Provide WDN hydraulic features.

(3) Calculate nodal load and capacity.

Step 2 (hazard information)

(1) Assess the ground motion parameters of WDN components under seismic scenario.

(2) Calculate the failure probability of each component.

Step 3 (cascading failure)

(1) Take one node in WDN as the initial failure node. Simulate the network topology after flow redistribution.

(2) If no new failure nodes are found, the cascading failure process stops. 


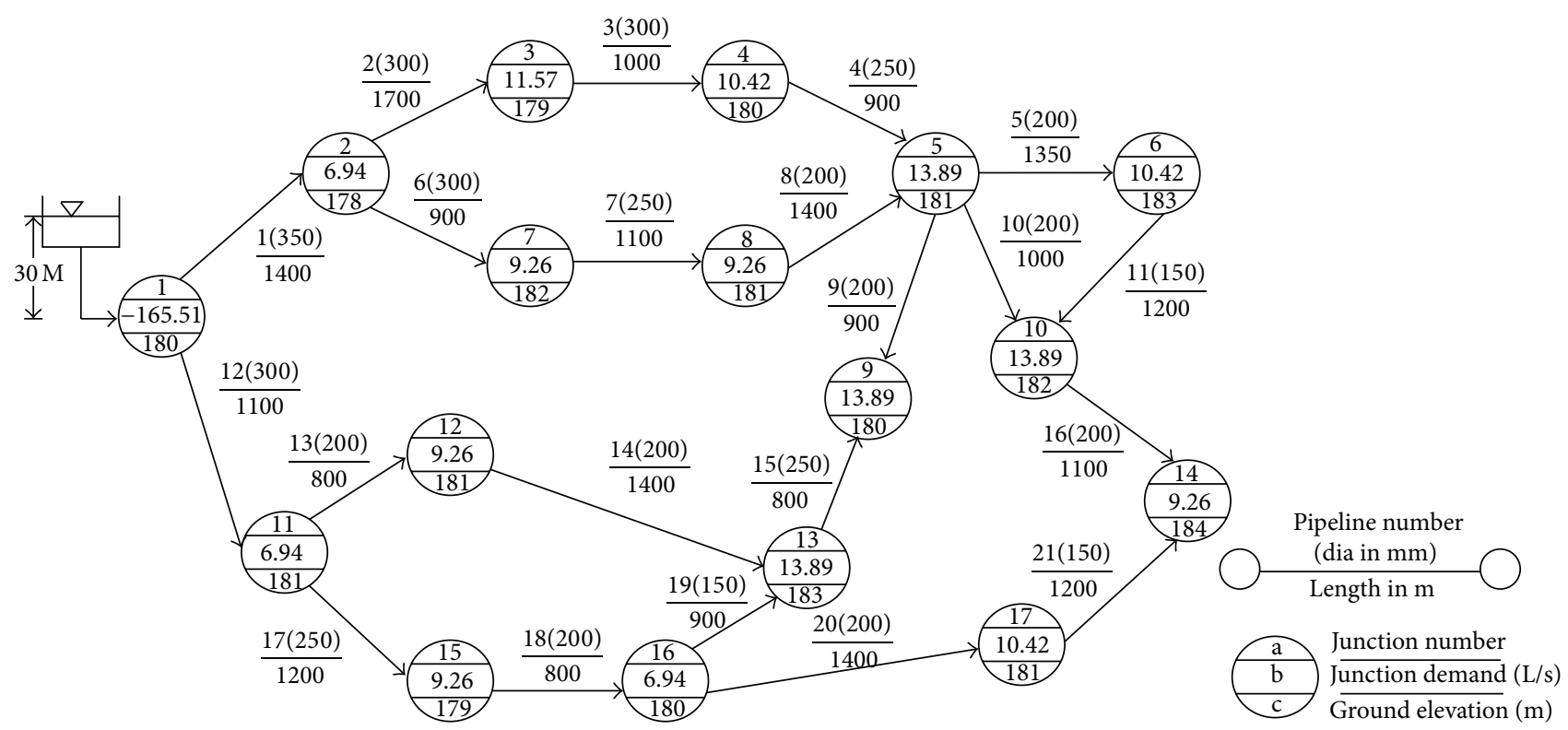

FIGURE 3: The layout of the WDN.

(3) Simulate the cascading failure process of each node successively. Conduct the system event vector.

Step 4 (serviceability)

(1) Calculate the system reliability according to the system event vector and failure probability.

(2) Evaluate the serviceability ratio using system reliability.

4.1. Hydraulic Analysis. Each operational node in WDN utilizes the laws of conservation of mass and energy to determine pressure and flow distribution throughout the network [26]. Formulations for water system are described as follows:

(1) For a junction node,

$$
\sum Q_{\text {in }}-\sum Q_{\text {out }}=q_{\text {ext }},
$$

where $Q_{\text {in }}$ and $Q_{\text {out }}$ are the inflow and outflow of the node; $q_{\text {ext }}$ is the external demand or supply.

(2) For any path,

$$
\sum_{i \in I_{p}} h_{L, i}+\sum_{j \in J_{p}} h_{p, j}=\Delta E
$$

where $h_{L, i}$ is the head loss across component $i$ along the path, $h_{p, j}$ is the head added by pump $j$, and $\Delta E$ is the difference in energy between the end points of the path.

The friction $\left(h_{L}\right)$ in each pipe is calculated using the Hazen-Williams equations, given as

$$
h_{L}=10.654\left(\frac{Q}{C}\right)^{1.852} \frac{1}{D^{4.87}} L,
$$

where $Q$ is the flow rate; $C$ is the Hazen-Williams coefficient; $D$ is the pipe diameter; and $L$ is the pipe length.
(3) For closed loops that begin and end at the same point $(\Delta E=0)$,

$$
\sum_{i \in I_{L}} h_{L, i}=0
$$

4.2. Seismic Hazard. In order to measure the vulnerability of WDN components under seismic action, the paper takes a single seismic origin as the case and the earthquake magnitude is set as 7.0. The earthquake magnitude represents the strength of the earthquake. It is related to the energy released by the earthquake. The greater the release of energy is, the greater the magnitude of the earthquake will be. The peak ground acceleration (PGA) is used to propagate the earthquake intensity. PGA is equal to the maximum ground acceleration that occurred at a site during a particular earthquake. As the selection of attenuation function is not in the scope of this paper, the seismic intensity attenuation relation proposed by Toro et al. [27] is applied to describe the variation of PGA:

$$
\begin{aligned}
\ln (\mathrm{PGA})= & 2.20+0.81 \cdot\left(M_{w}-6.0\right)-1.27 \\
& \cdot \ln \left(\sqrt{R^{2}+9.3^{2}}\right)-(1.16-1.27) \\
& \cdot \max \left[\ln \left(\frac{\sqrt{R^{2}+9.3^{2}}}{100}\right), 0.0\right] \\
& -0.0021 \sqrt{R^{2}+9.3^{2}}+\ln (1.52),
\end{aligned}
$$

where $M_{w}$ is the earthquake magnitude. The earthquake magnitude is assumed to 7.0. $R$ is the distance between the site of interest (water node) and the epicenter. The distances of Nodes 1-17 in the network are 37.189, 37.804, 38.079, 38.075, $37.487,37.693,37.350,37.323,36.864,36.666,36.592$, 36.754, $36.560,35.567,35.583,35.881$, and $35.531 \mathrm{~km}$, respectively. 
TABLE 1: The cascading failure processes ( $\alpha=0.5$ and $\alpha=1.0)$.

\begin{tabular}{lcc}
\hline Node & Failure process $(\alpha=0.5)$ & Failure process $(\alpha=1.0)$ \\
\hline 1 & All nodes failed & All nodes failed \\
2 & $2 \rightarrow(3-10,13,14,17) \rightarrow(12,16)$ & $2 \rightarrow(3-10,13,14,17) \rightarrow(16)$ \\
3 & $3 \rightarrow(4,5,6,9,10,13,14,17) \rightarrow(8,12,16)$ & $3 \rightarrow(4,5,6,9,10,13,14,17) \rightarrow(16)$ \\
4 & $4 \rightarrow(3,5,6,9,10,13,14,17) \rightarrow(8,12,16)$ & $4 \rightarrow(3,5,6,9,10,13,14,17) \rightarrow(16)$ \\
5 & $5 \rightarrow(4,6,8,9,10,13,14,17) \rightarrow(12,16)$ & $5 \rightarrow(4,6,9,10,13,14,17) \rightarrow(3,16)$ \\
6 & $6 \rightarrow(5,9,10,14,17) \rightarrow(4,8,13,16) \rightarrow(12)$ & $6 \rightarrow$ no more failure nodes \\
7 & $7 \rightarrow(8,14) \rightarrow(5,6,9,10,13,16,17) \rightarrow(4,12)$ & $7 \rightarrow(8,14) \rightarrow(6,10,17)$ \\
8 & $8 \rightarrow(7,14) \rightarrow(5,6,9,10,13,16,17) \rightarrow(4,12)$ & $8 \rightarrow(7,14) \rightarrow(6,10,17)$ \\
9 & $9 \rightarrow(5,6,10,13,14,16,17) \rightarrow(4,8,12)$ & $9 \rightarrow(6,14) \rightarrow(5,10,17) \rightarrow(4) \rightarrow(3)$ \\
10 & $10 \rightarrow(5,6,9,14) \rightarrow(4,8,13,16,17) \rightarrow(12)$ & $10 \rightarrow(6,14) \rightarrow(17)$ \\
11 & $11 \rightarrow(6,10,12-17) \rightarrow(4,5,9) \rightarrow(3,8)$ & $11 \rightarrow(6,10,12-17)$ \\
12 & $12 \rightarrow(13,14) \rightarrow(5,6,9,10,16,17) \rightarrow(4,8)$ & $12 \rightarrow(13,14) \rightarrow(6,10,17) \rightarrow(16)$ \\
13 & $13 \rightarrow(12,14,16,17) \rightarrow(5,6,9,10) \rightarrow(4,8)$ & $13 \rightarrow(12)$ \\
14 & $14 \rightarrow(5,6,9,10,13,16,17) \rightarrow(4,8,12)$ & $14 \rightarrow(6,10,17)$ \\
15 & $15 \rightarrow(14,16,17) \rightarrow(5,6,9,10,13) \rightarrow(4,8,12)$ & $15 \rightarrow(14,16,17) \rightarrow(6,10)$ \\
16 & $16 \rightarrow(14,15,17) \rightarrow(5,6,9,10,13) \rightarrow(4,8,12)$ & $16 \rightarrow(14,15,17) \rightarrow(6,10)$ \\
17 & $17 \rightarrow(13,16) \rightarrow(6,10,12,14) \rightarrow(4,5,9) \rightarrow(3,8)$ & $17 \rightarrow$ no more failure nodes \\
\hline
\end{tabular}

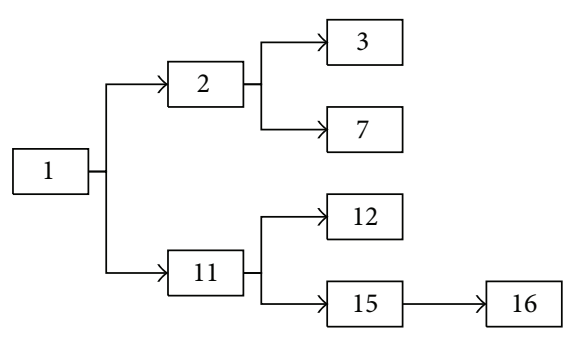

(a) $t=2$

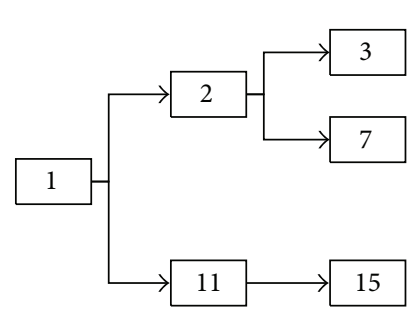

(b) $t=3$

FIgURE 4: The state of WDN after Node 5 failed.

Structural fragility curves are essential for evaluation of the functionality of water distribution component under earthquake impact. The structural fragility in Adachi and Ellingwood [28] is used. The fragility of each component in the WDN is described by the lognormal cumulative distribution function (CDF):

$$
P f_{x}(x)=\int_{0}^{x} \frac{1}{\sqrt{2 \pi} \xi s} \exp \left[-\frac{1}{2}\left\{\frac{\ln (s)-\lambda}{\zeta}\right\}^{2}\right] d s,
$$

where $s$ is the ground motion intensity and $\lambda$ and $\xi$ are the median and standard deviation of the lognormal distribution, respectively. The fragilities are defined by median of $0.4 \mathrm{~g}$ and logarithmic standard deviation of 0.4 [29].

4.3. Cascading Failure Process. The cascading failure processes of WDN have been simulated with $\alpha=0.5$ and $\alpha=1.0$. The failure processes are shown in Table 1 . It can be found that the number of secondary failure nodes has a significant reduction when the constraint condition of node bearing capacity is relaxed. As $\alpha=0.5$ and 1.0, the numbers of failure nodes among Nodes $2,3,4,7,8,11,12,15$, and 16 at $t=2$ are the same; as $t \geq 3$, the failure scale of $\alpha=0.5$ is larger than that of $\alpha=1.0$. For other nodes, for example, Nodes 5, 6, 7, $10,13,14$, and 17, the failure scale of $\alpha=0.5$ is larger than that of $\alpha=1.0$ at $t=2$. This is obvious for Node 6 and Node 17: as $\alpha=0.5$, the nodal failure of Node 6 and Node 17 leads to the extra 10 and 11 failure nodes, respectively; as $\alpha=1.0$, the nodal failure of Node 6 and Node 17 cannot trigger the cascading failure. The cascading failure simulation is used to generate the connected link, which can be used to conduct the system event vector.

4.4. System Reliability. Take Node $5(\alpha=0.5)$ as an example. Figure 4 shows the connected state at $t=2$ and $t=3$. Node 5 is damaged under seismic action and its failure process can be divided into three stages. At $t=1$, Node 5 fails under the earthquake and its failure leads to the failure of its connected pipes which further results in the redistribution of WDN; at $t=2$, Nodes 4, 6, 8, 9, 10,13,14, and 17 fail successively; at $t=$ 3 , Nodes 12 and 16 fail; then the system reaches a new stable state; that is, no new failure node occurs. The effective event vectors of supply node $c^{E_{i}}(i=1,2, \ldots, 17)$ are constructed by (3a), (3b), (3c), and (5). 


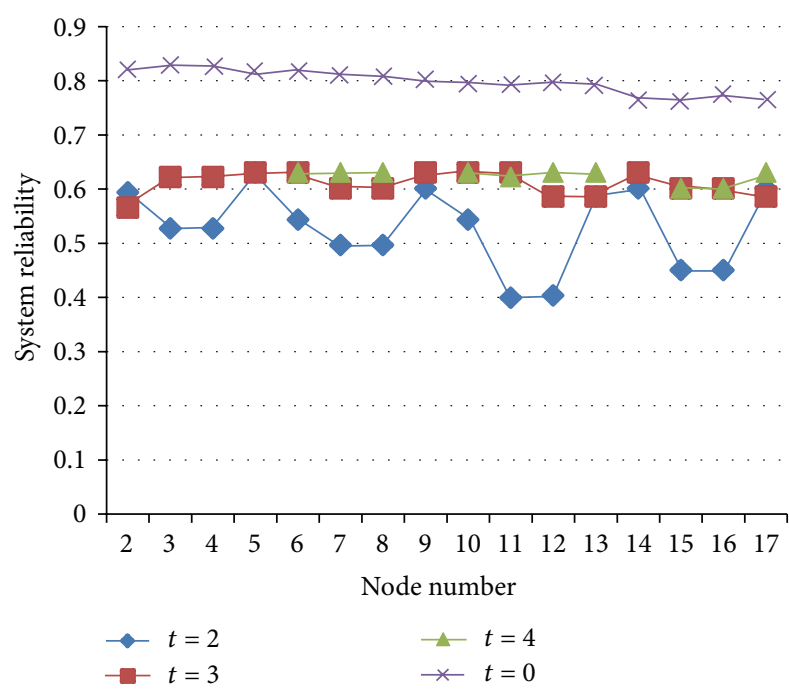

(a) $\alpha=0.5$

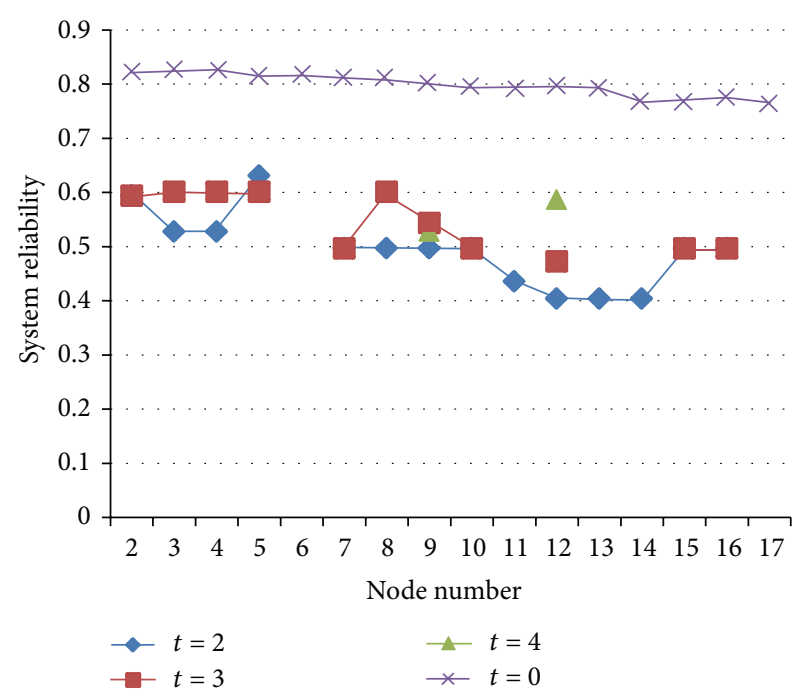

(b) $\alpha=1.0$

FIGURE 5: Computed results of the system reliability at each time.

At $t=2$, Nodes $4,6,8,9,10,13,14$, and 17 fail and the remaining connected nodes are Nodes $1,2,3,7,11,12$, 15, and 16. The system event vector can be expressed as

$$
\begin{aligned}
E_{\text {sys }}^{5-\mathrm{II}}=E_{1} & \cap\left\{\left[E_{2} \cap\left(E_{3} \cup E_{7}\right)\right]\right. \\
\cup & {\left.\left[E_{11} \cap\left(E_{12} \cup\left(E_{15} \cap E_{16}\right)\right)\right]\right\} . }
\end{aligned}
$$

At $t=3$, Nodes 12 and 16 fail. The remaining connected nodes are Nodes $1,2,3,7,11$, and 15 . The system event vector can be expressed as

$$
E_{\text {sys }}^{5 \text {-III }}=E_{1} \cap\left\{\left[E_{2} \cap\left(E_{3} \cup E_{7}\right)\right] \cup\left[E_{11} \cap E_{15} \cap E_{16}\right]\right\} .
$$

Similarly, by the change of network topology after the cascading failure occurred, we can construct the subsystem event vector constituted by the remaining work nodes at each time. After the supply Node 1 fails, Nodes 2-17 are bound to fail in the absence of other supplies, so the supply node is the most critical node. Here, we focus on Nodes $2-17$ in the influence of cascading failure and seismic action.

Simulations are made successively with Nodes 2-17 failure. Construct the connected graph of the remaining work nodes at each time. Calculate the functionality of the system under seismic action by (13). The subsystem event is constituted by the remaining work nodes after cascading failure occurs. Different failure nodes lead to different remaining nodes which constitute different subsystems. Consider the spatial correlation of the nodes; use LP method (4) to calculate the boundary of system reliability probability. Define the average of the supremum and infimum boundary as the system reliability.

Figure 5 shows the trendline of the system reliability at different time. In Figure 5, the purple line represents the reliability of the single node under seismic action. The blue line, red line, and green line represent the subsystem reliability at $t=2,3$, and 4 , respectively. The system reliability is influenced by three aspects: (1) the distance from network components to the epicenter; (2) the functionality of nodes; and (3) the change of the network topology. The change of the network topology is obtained from the constraints conditions of load and capacity. First of all, it can be seen that the system reliability is lower than that of the components. Secondly, as shown in Figure 5(a), the fluctuation of system reliability is obvious; this is mainly because the small value of $\alpha$ and the low upper-limit of bearing capacity lead to the enlargement of unconnected scope. Thirdly, when $\alpha=0.5$, Node 2's system reliability reduces at $t=3$ which means Node 2 corresponds to the parallel position. Other nodes at each time are close to the series position with an increased system reliability. When $\alpha=1.0$, Node 5 at $t=3$ and Node 9 at $t=4$ are close to the parallel position. Other nodes at each time are close to the series position.

4.5. Serviceability. Based on the efficiency analysis of the subsystem under seismic action, the serviceability of WDN is analyzed also. Consider the weight factor $\omega$ as the pipe length $L$. The computed result is shown in Figures 6 and 7.

Figure 6 illustrates the serviceability ratio at each time step under different tolerance parameters $(\alpha=0.5$ and $\alpha=$ 1.0). In Figure 6, the blue bar, red bar, and green bar represent the serviceability ratio at $t=2,3$, and 4 , respectively. As the process of cascading failure, earthquake, and service area have been taken into consideration, the result shows that, with the progress of cascading failure, the remaining serviceability is gradually declining while the service failure is increasing. There is a great difference between $t=2$ and $t=3$, indicating that the first secondary failures caused by the failure of initial node lead to a significant decline of serviceability. Therefore, it is necessary to control the cascading failure between $t=2$ and $t=3$.

Specific to $t=2$ and $t=3$, Figure 7 shows the difference on serviceability ratio. In Figure 7, the blue line and the red 


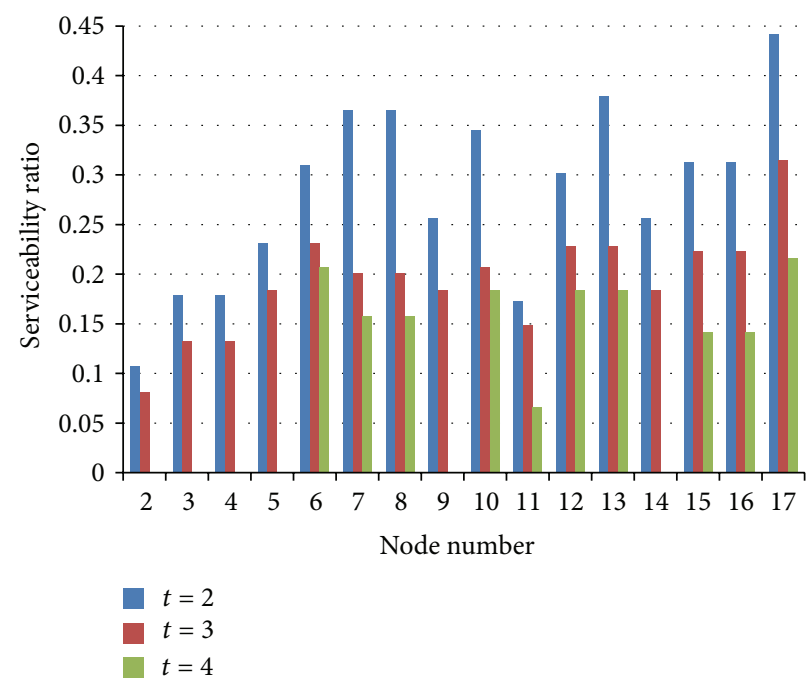

(a) $\alpha=0.5$

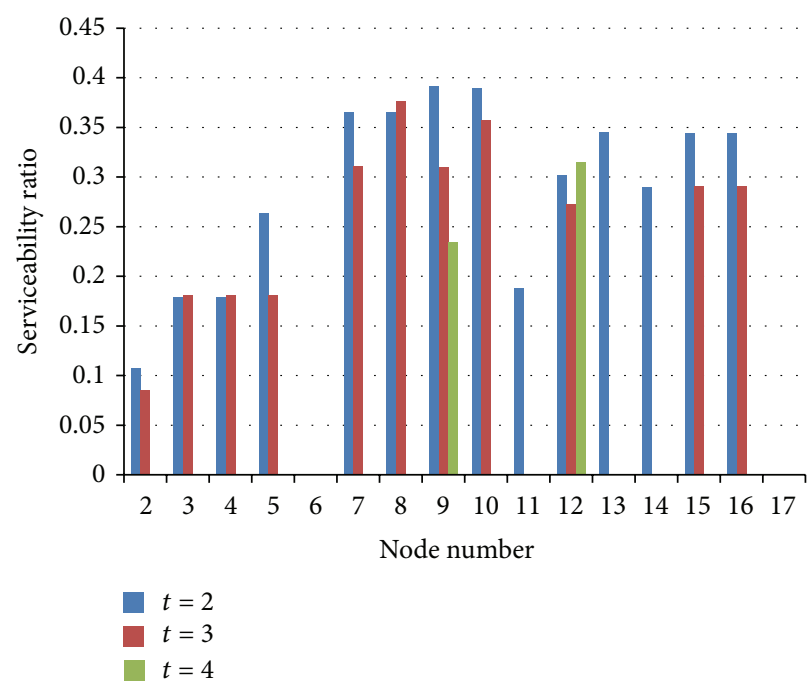

(b) $\alpha=1.0$

FIGURE 6: Result of the serviceability ratio $\left(M_{w}=7 ; \alpha=0.5,1.0\right)$.

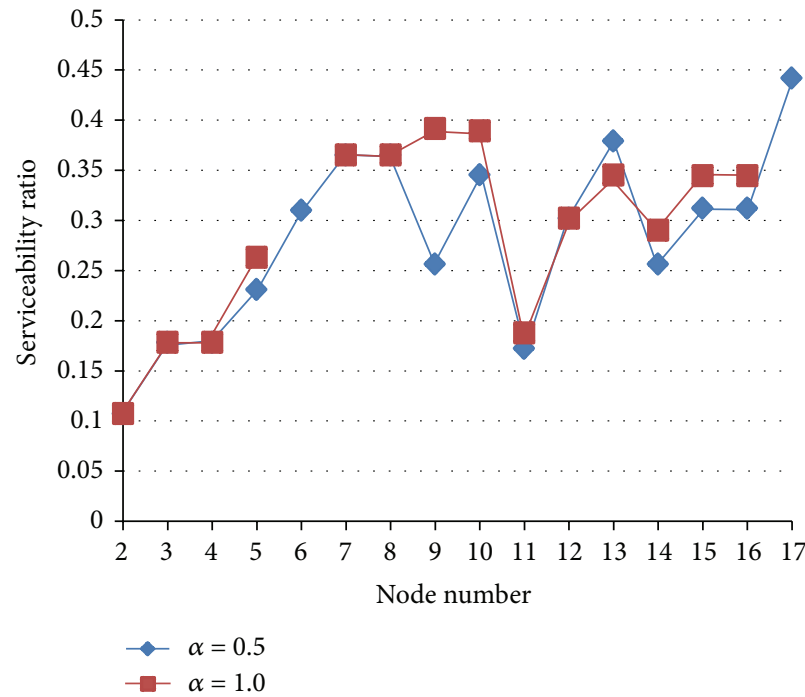

(a) $t=2$

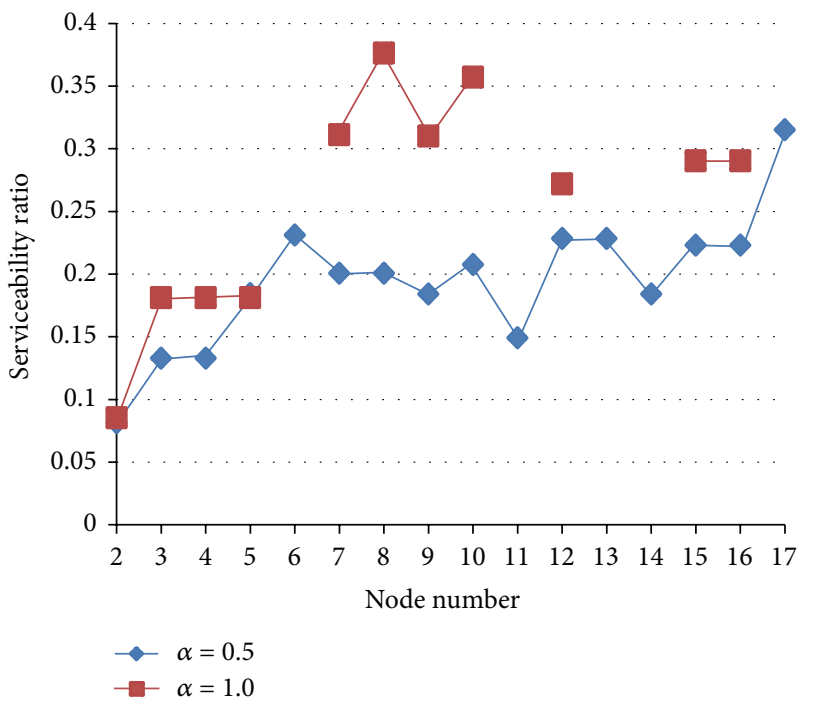

(b) $t=3$

FIGURE 7: Serviceability ratio with $t=2$ and $t=3(\alpha=0.5 ; \alpha=1.0)$.

line represent the serviceability ratio under $\alpha=0.5$ and $\alpha=1.0$, respectively. The serviceability when $\alpha=1.0$ is higher than or equal to those when $\alpha=0.5$. This is mainly because the maximum pressure constraint is more relaxed as $\alpha=1.0$. It should be noted that the serviceability ratio of Node 2 remains unchanged. In Figure 7(a), Nodes 2, 3, 4, 7, 8 , and 12 have the same serviceability ratio at $t=2$ under $\alpha=0.5$ and $\alpha=1.0$. In Figure 7(b), Nodes 2 and 5 have the same serviceability ratio at $t=3$ under $\alpha=0.5$ and $\alpha=1.0$. The intersection of Figures 7(a) and 7(b) is Node 2. It is thus clear that the serviceability of Node 2 under any tolerance parameter always is the minimum one, which indicates that Node 2 is the most critical node. A backup device is needed to Node 2 to enhance the antidisaster ability of the system.

\section{Conclusion}

To evaluate the serviceability of WDN under seismic scenario, the component functionality and the cascading failures are taken into consideration. The serviceability is measured in three aspects: (1) functionality of structural components under disaster environment; (2) recognition of cascading failure process, that is, the connectivity loss between nodelinks; and (3) calculation of the subsystem reliability. Results show that the topological position of the failure nodes can be measure by the subsystem reliability. The critical node can be identified. Secondly, under different values of $\alpha$, the serviceability of the subsystem presents a great difference which can be attributed to the different failure processes 
under cascade effect. Thirdly, it is necessary to control the cascading failure between $t=2$ and $t=3$. Conducting timely repair and maintenance to WDN before and during this period can enhance the antidisaster ability of WDN.

Further research should be focused on the time. Time $t$ is used as an algorithmic step. Future works should focus on the real meaning of time; that is, the cascading failure step can be combined with flow velocity to express the hours, minutes, and seconds. Also, a time constraint can be added in the model to evaluate the dynamic relationship on the serviceability, the failure time, and the repair time. Besides, there is a reduction level of water nodes after the damage. The reduction level of service should be extended in the model to obtain a more realistic result.

\section{Competing Interests}

The authors declare that they have no competing interests.

\section{Acknowledgments}

This work was funded by the National Natural Science Foundation of China under Grant no. 71501008, the Fundamental Funds for Humanities and Social Sciences of Beijing Jiaotong University (no. 2015jbwj014), and the Fundamental Funds of Beijing Jiaotong University under Grant (no. 2015RC002).

\section{References}

[1] A. Yazdani and P. Jeffrey, "Complex network analysis of water distribution systems," Chaos, vol. 21, Article ID 016111, 2011.

[2] J. Yates and S. Sanjeevi, "Assessing the impact of vulnerability modeling in the protection of critical infrastructure," Journal of Geographical Systems, vol. 14, no. 4, pp. 415-435, 2012.

[3] T. Adachi, Impact of cascading failures on performance assessment of civil infrastructure systems [Ph.D. thesis], Georgia Institute of Technology, Atlanta, Ga, USA, 2007.

[4] L. Dueñas-Osorio and S. M. Vemuru, "Cascading failures in complex infrastructure systems," Structural Safety, vol. 31, no. 2, pp. 157-167, 2009.

[5] C.-L. Chai, X. Liu, W. J. Zhang, and Z. Baber, "Application of social network theory to prioritizing Oil \& Gas industries protection in a networked critical infrastructure system," Journal of Loss Prevention in the Process Industries, vol. 24, no. 5, pp. 688694, 2011.

[6] K. J. Tierney, S. E. Chang, and R. T. Eguchi, Improving Earthquake Loss Estimation: Review, Assessment and Extension of Loss Estimation Methodologies, University of Delaware. Disaster Research Center, 1999.

[7] M. Shinozuka, H. Hwang, and M. Murata, "Impact on water supply of a seismically damaged water delivery system," in Proceeding of the Lifeline Earthquake Engineering in the Central and Eastern US, pp. 43-57, ASCE, September 1992.

[8] H. H. M. Hwang, H. Lin, and M. Shinozuka, "Seismic performance assessment of water delivery systems," Journal of Infrastructure Systems, vol. 4, no. 3, pp. 118-125, 1998.

[9] T. Adachi and B. R. Ellingwood, "Serviceability of earthquakedamaged water systems: effects of electrical power availability and power backup systems on system vulnerability," Reliability Engineering \& System Safety, vol. 93, no. 1, pp. 78-88, 2008.
[10] S. A. Brink, R. A. Davidson, and T. H. P. Tabucchi, "Strategies to reduce durations of post-earthquake water service interruptions in Los Angeles," Structure and Infrastructure Engineering, vol. 8, no. 2, pp. 199-210, 2012.

[11] T. H. Grubesic and A. T. Murray, "Vital nodes, interconnected infrastructures, and the geographies of network survivability," Annals of the Association of American Geographers, vol. 96, no. 1, pp. 64-83, 2006.

[12] A. E. Motter and Y.-C. Lai, "Cascade-based attacks on complex networks," Physical Review E, vol. 66, Article ID 065102, 2002.

[13] P. Crucitti, V. Latora, and M. Marchiori, "Model for cascading failures in complex networks," Physical Review E-Statistical, Nonlinear, and Soft Matter Physics, vol. 69, no. 4, Article ID 045104, 2004.

[14] Y. Wang and S.-K. Au, "Spatial distribution of water supply reliability and critical links of water supply to crucial water consumers under an earthquake," Reliability Engineering \& System Safety, vol. 94, no. 2, pp. 534-541, 2009.

[15] J. Nigg, "Perceptions of earthquake impacts and loss-reduction policy preferences among community residents and option leaders," Disaster Research Center, University of Delaware, 1998.

[16] J.-W. Wang and L.-L. Rong, "Cascade-based attack vulnerability on the US power grid," Safety Science, vol. 47, no. 10, pp. 13321336, 2009.

[17] J. Wang, C. Jiang, and J. Qian, "Robustness of Internet under targeted attack: a cascading failure perspective," Journal of Network and Computer Applications, vol. 40, no. 1, pp. 97-104, 2014.

[18] J. Wang, "Robustness of complex networks with the local protection strategy against cascading failures," Safety Science, vol. 53, pp. 219-225, 2013.

[19] J.-W. Wang and L.-L. Rong, "Robustness of the western United States power grid under edge attack strategies due to cascading failures," Safety Science, vol. 49, no. 6, pp. 807-812, 2011.

[20] J. Song and A. D. Kiureghian, "Bounds on system reliability by linear programming," Journal of Engineering Mechanics, vol. 129, no. 6, pp. 627-636, 2003.

[21] W.-H. Kang, J. Song, and P. Gardoni, "Matrix-based system reliability method and applications to bridge networks," Reliability Engineering \& System Safety, vol. 93, no. 11, pp. 1584-1593, 2008.

[22] J. Song and W.-H. Kang, "System reliability and sensitivity under statistical dependence by matrix-based system reliability method," Structural Safety, vol. 31, no. 2, pp. 148-156, 2009.

[23] D. Bertsimas and J. Tsitsiklis, Introduction to Linear Optimization, Athena Scientific Press, 1st edition, 1997.

[24] H.-W. Lim and J. Song, "Efficient risk assessment of lifeline networks under spatially correlated ground motions using selective recursive decomposition algorithm," Earthquake Engineering \& Structural Dynamics, vol. 41, no. 13, pp. 1861-1882, 2012.

[25] M. L. Kansal, A. Kumar, and P. B. Sharma, "Reliability analysis of water distribution systems under uncertainty," Reliability Engineering and System Safety, vol. 50, no. 1, pp. 51-59, 1995.

[26] L. W. Mays, Water Distribution System Handbook, McGraw-Hill Professional Publishing, New York, NY, USA, 1999.

[27] G. R. Toro, N. A. Abrahamson, and J. F. Schneider, "Model of strong ground motions from earthquakes in central and eastern North America: best estimates and uncertainties," Seismological Research Letters, vol. 68, no. 1, pp. 41-57, 1997. 
[28] T. Adachi and B. R. Ellingwood, "Comparative assessment of civil infrastructure network performance under probabilistic and scenario earthquakes," Journal of Infrastructure Systems, vol. 16, no. 1, Article ID 010001QIS, pp. 1-10, 2010.

[29] FEMA, "HAZUS MH MR1," Technical Manual, Washington, DC, USA, 2003. 


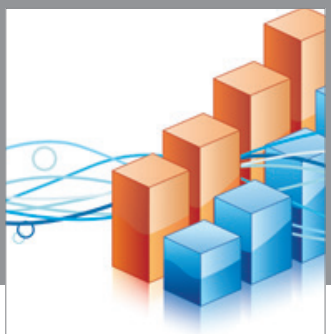

Advances in

Operations Research

vatem alat4

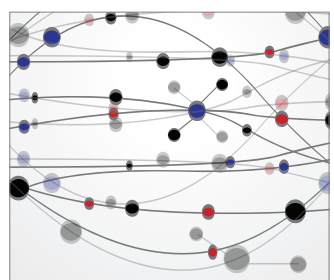

\section{The Scientific} World Journal
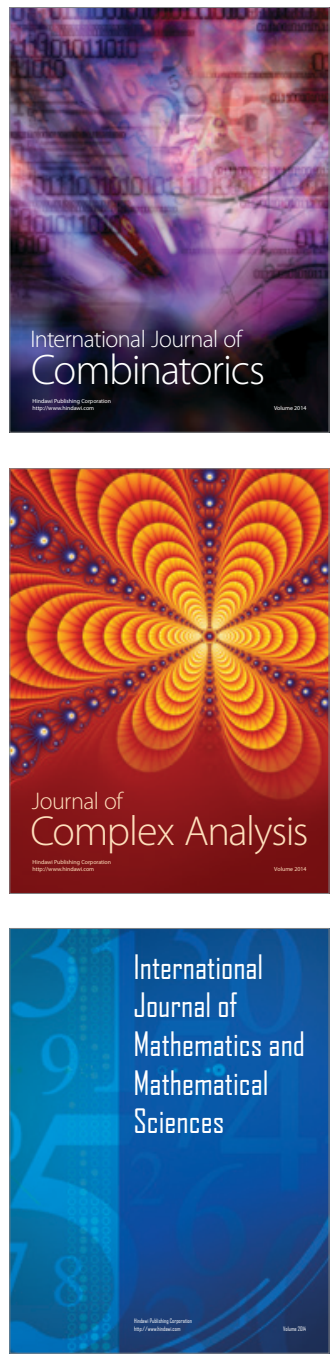
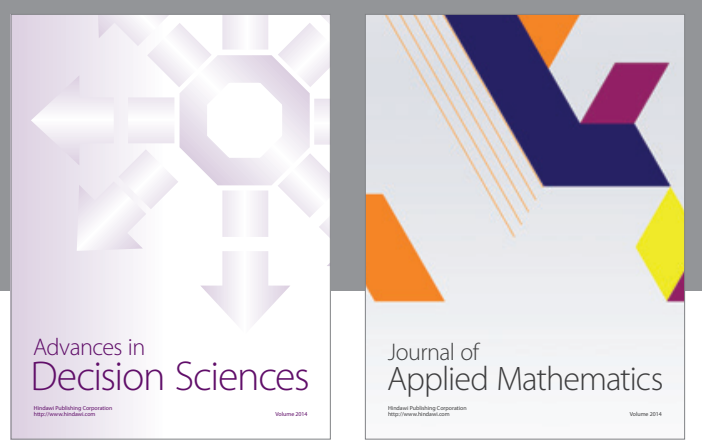

Algebra

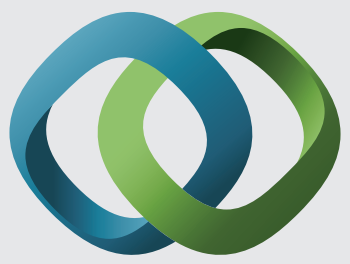

\section{Hindawi}

Submit your manuscripts at

http://www.hindawi.com
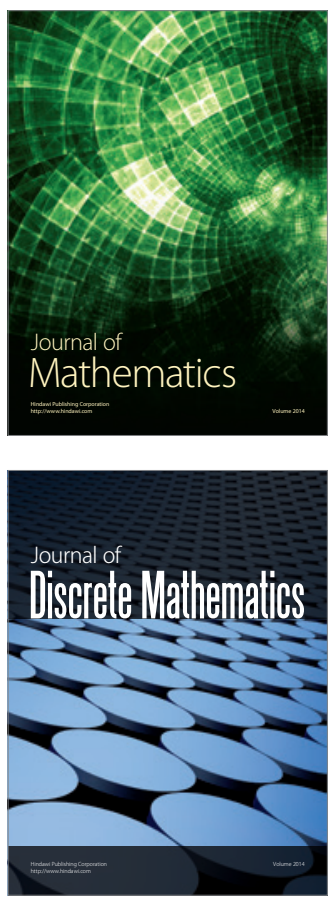

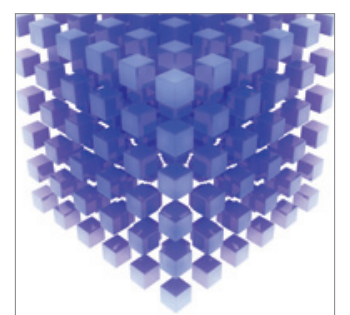

Mathematical Problems in Engineering
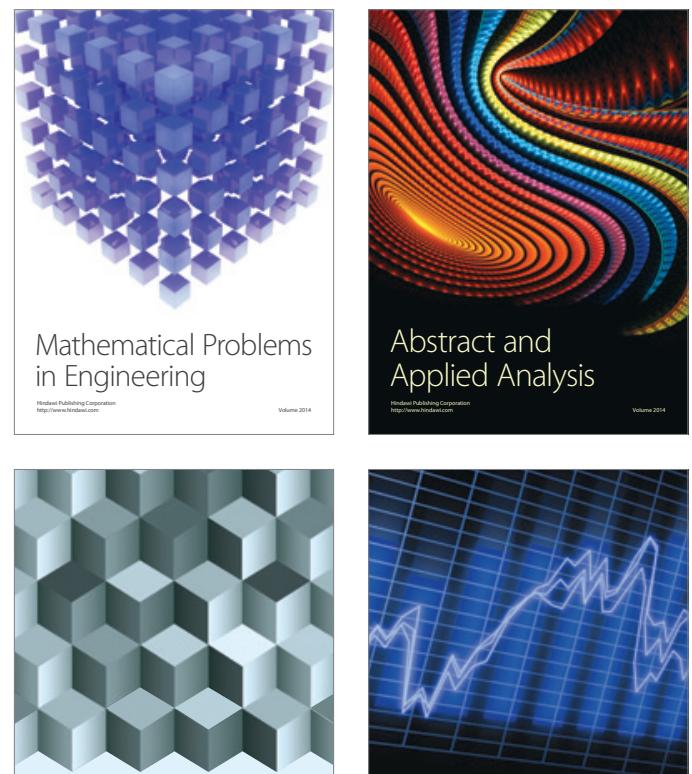

Journal of

Function Spaces

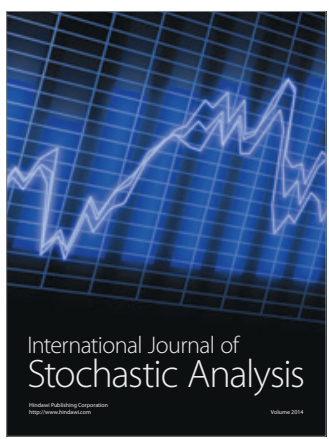

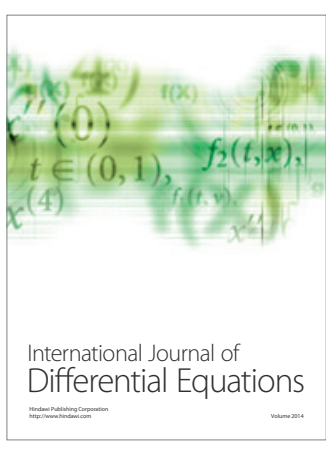
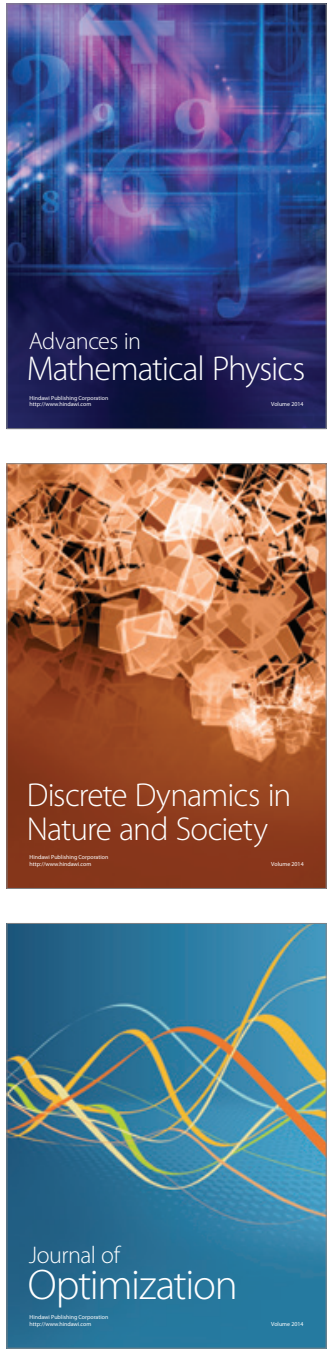